\title{
BP-VE-3
}

\section{Laparoscopic management of post pancreaticoduodenectomy benign hepaticojejunostomy stricture: A case report}

\author{
Shankar BALASUBRAMANIAN*, Selvakumar BALAKRISHNAN, Palanivelu CHINNUSAMY \\ Department of HPB and Minimal Access Surgery, Gem Hospital \& Research Center, Chennai, India
}

Introduction: Hepaticojejunostomy (HJ) stricture following pancreaticoduodenectomy (PD), though rare, can have serious consequences as they can present with recurrent cholangitis. Percutaneous interventional procedures are the usual first line treatment options but have high re-stricture rates and require multiple sittings.

Methods: We present a case of 64 year old gentleman who underwent open PD for ampullary adenocarcinoma in 2010 and received 6 cycles of adjuvant chemotherapy. He had recurrent attacks of cholangitis since June 2019 and was managed initially with medical therapy. He was diagnosed to have a stricture of $\mathrm{HJ}$ with multiple stones in the biliary system proximally. After failed conservative therapy, the patient was subjected to laparoscopic revision of HJ. After adhesiolysis, the previous HJ was dismantled, ducts cleared off stones and an end to side HJ was done using PDS 3-0 sutures.

Results: The total operating time was 165 minutes and the total blood loss was about $100 \mathrm{~mL}$. The patient developed fever on post-operative day (POD) 2 and was managed with antibiotics. He was discharged on POD 6. He has been on follow-up for more than three months now and has been asymptomatic.

Conclusions: Laparoscopic revision of HJ stricture following PD appears to be feasible with good outcome when performed by an experienced hepatobiliary surgeon. Long term follow-up is needed before firm conclusions can be drawn. 